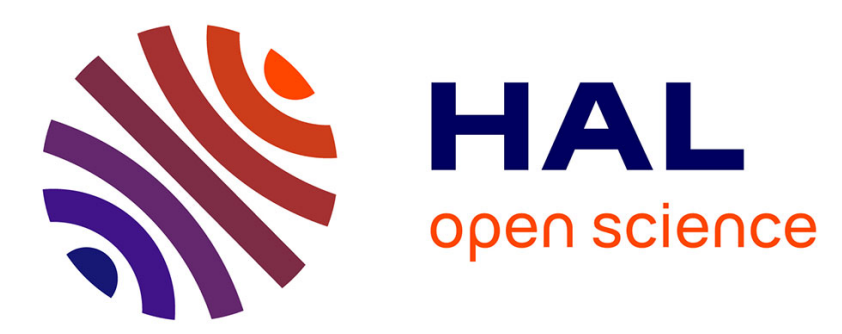

\title{
High-altitude free fall and parameter estimation for undergraduate numerical techniques laboratory.
}

Francois Lehmann

\section{To cite this version:}

Francois Lehmann. High-altitude free fall and parameter estimation for undergraduate numerical techniques laboratory.. European Journal of Physics, 2020, 41 (5), pp.055803. 10.1088/13616404/ab9dc4 . hal-03015626

\section{HAL Id: hal-03015626 \\ https://hal.science/hal-03015626}

Submitted on 19 Nov 2020

HAL is a multi-disciplinary open access archive for the deposit and dissemination of scientific research documents, whether they are published or not. The documents may come from teaching and research institutions in France or abroad, or from public or private research centers.
L'archive ouverte pluridisciplinaire HAL, est destinée au dépôt et à la diffusion de documents scientifiques de niveau recherche, publiés ou non, émanant des établissements d'enseignement et de recherche français ou étrangers, des laboratoires publics ou privés. 
2 High-altitude free fall and parameter estimation for undergraduate numerical techniques laboratory.

3 François LEHMANN

4 Laboratoire d'Hydrologie et Géochimie de Strasbourg, Université de Strasbourg/EOST/ENGEES, CNRS, 51 rue Blessig 67084 Strasbourg, France.

6

7 First draft April - Mai 2020,

8

Accepted Manuscript online 17 June 2020, DOI https://doi.org/10.1088/1361-6404/ab9dc4

\section{Abstract}

In this article, we present the development and treatment of an inverse problem applied to data from the Felix Baumgartner stratospheric jump. This jump is well documented with a lot of data. Therefore, it makes a particularly well-suited example for teaching in a numerical techniques laboratory. The major aim of the article is to give guidelines in order to construct a simple, but not simplistic, inverse problem with real data for junior undergraduate students. Students should master classical mechanics and have some skills in numerical modelling. We use the programming language Python and various libraries in order to build a model and solve the entire problem. This programming language is increasingly used and understood by students, which allows them to focus on the physical and numerical aspects of the involved problem. The fairly new strategy presented in this article is an attempt to estimate the angle of attack from acceleration measurements, and to give an uncertainty estimation of Baumgartner's free-fall speed. 
"Oh no, not the free-fall again!" Most first-ever physics courses start with classical mechanics, and especially with the study of the motion of solids. On 14 October 2012, the Red Bull Stratos team [1] shared with the world an extraordinary experiment, a leap from a capsule suspended $40 \mathrm{~km}$ above Earth, over Roswell, New Mexico. During this jump, the skydiver Felix Baumgartner was the first human to break the sound barrier without any thrust force. He maintained for a total of 30 seconds a speed greater than the speed of sound in air. Even many years later, the extraordinary experimental conditions of this free fall still make it a veritable 'playground' for any physicist who wants to teach classical mechanics and numerical modelling. Like any physics problem under extreme conditions, this experiment is more complex than it seems. It is a problem involving several scientific fields: mechanics, fluid dynamics, thermodynamics, numerical method and many others, such as pathophysiology or data acquisition. Anyone can understand it very quickly by reading the Red Bull report [2]. This is probably the reason why it makes a particularly well-suited example for teaching. It allows students to make connections between different scientific fields, to break the disciplinary boundaries and to learn to work as a team on a small project. This example could be realized by undergraduate students after a set of laboratory classes, such as that proposed by Samsonau [3]. To carry out this work in their third year, sophomore college students should have followed an introductory course in Python language, with an emphasis on applications in the physical sciences and engineering, on basic problem solving, programming techniques, and fundamental algorithms. Students who have experience in programming with Python of about 7 hours per week (1:30 lectures, 4 h computer laboratory time and 1:30 personal work) for 6 to 7 weeks can easily tackle this problem.

Modelling such an experiment is difficult given the non-linearity of the processes in an environment where air pressure, air temperature and air density are interrelated, and change rapidly with altitude. The mechanistic approach, which consists of describing phenomena in the context of the laws of conservation, is the most commonly used approach. It constitutes a powerful tool for understanding and seems best suited for predictive simulations. However, we should also not forget that the key to the modelling approach is to build a model that is simple, realistic and feasible. The most commonly used approach for modelling such a high-altitude free fall is to use Newton's second law to consider gravitational force, and an aerodynamic force that opposes the skydiver's motion through the air. This model has been used by many authors [4 - 8] in order to estimate the position and velocity of the skydiver. An excellent introduction for students to this kind of problem can be found in the book written by Barger and Olsson [9]. This paper can be seen as a continuation of the earlier works of Colino and Barbero [6] and Guerster and Walter [7]. 
In order to build the model, we need to completely define the forces included in the equation of motion, consisting of all the parametric functions and the initial conditions. Most of the time, not all the parameters are well known. Sometimes, we don't know the exact value of a specific parameter. Some of these parameters cannot be directly measured, so in such conditions we can use parameter estimation techniques in order to estimate the parameter values and also, importantly, the parameter uncertainty and ultimately, calculating the associated uncertainty of the computed position and velocity of the skydiver, whilst taking into account the available measures.

This paper follows a three-fold structure: first, building the direct problem in which the physical system of the free fall is modeled, with all parameters and with the initial conditions. Second, the inverse problem is merged with the direct problem and the data. Finally, the last section illustrates the global model application to determine an estimation of the parameters and their associated uncertainties.

\section{The direct problem}

In order to establish the equation of motion, Newton's second law is applied to a body of mass $\mathrm{m}$ subject to two forces, gravitational force $\vec{P}$ and drag force $\overrightarrow{F_{D}}$ :

$$
m \vec{a}=\vec{P}+\overrightarrow{F_{D}}
$$

$\vec{a}$ is the body acceleration in $\mathrm{m} \mathrm{s}^{-2}$. If the free fall is strictly vertical and the positive $\mathrm{z}$ direction is chosen to be up, the equation of motion, as described in detail by Guerster and Walter [7], is given by:

$$
m \frac{d v_{z}}{d t}=-m g(z)+\frac{1}{2} C_{D}(M a) A_{\perp}(\beta) \rho(z, T) v_{z}^{2}
$$

where $C_{D}(M a)$ is the drag coefficient, which depends on the Mach number $\mathrm{Ma}, A_{\perp}$ is the projected area depending on the angle of attack $\beta$ (the angle between the $z$ axis reversed and a longitudinal reference line on the body, head first), $\rho(z, T)$ the air density depending on altitude $z$ and air temperature $\mathrm{T}, g(z)$ the acceleration due to gravity depending on $z$. For this equation of motion, the state variables are the altitude $z$ and the speed $v_{z}$. The aim of the direct problem is to compute the state variables, which are dependent variables, against time (the independent variable). This can be done by rewriting the second-order differential equation (2) as a system of two first-order equations:

$$
\left\{\begin{array}{c}
\frac{d z}{d t}=v_{z} \\
\frac{d v_{z}}{d t}=-g(z)+\frac{1}{2 m} C_{D}(M a) A_{\perp}(\beta) \rho(z, T) v_{z}^{2}
\end{array}\right.
$$

To determine the evolution of the body, it is necessary to define its initial state, that is the initial position and the initial velocity. At this step, it is also necessary to define all the parametric functions which complete the model. The same relations as those used by Guerster and Walter [7] are included within the model. 
Before defining explicitly all the parametric functions, it is important to fix the geographical coordinate system in which the trajectory is studied. The initial GPS position ( $\varphi$ :latitude, $\lambda$ :longitude, z:altitude), when Baumgartner left the capsule on 14 October 2012, at 18:06:32.0 UTC, was ( $\varphi=33.3408417^{\circ}$, $\left.\lambda=-103.7679067^{\circ}, z=38969.4 \mathrm{~m}\right)$. To study the trajectory and the speed of Baumgartner during his fall, we choose a local tangent plane coordinates system with an origin given by $(\varphi=$ $33.3408417^{\circ}, \lambda=-103.7679067^{\circ}, z=0.0 \mathrm{~m}$ ); by convention the east axis is labeled $x_{\text {East }}$, the north $y_{N o r t h}$ and the up $z_{U p}$. In this local ENU (East, North, Up) coordinate system, the trajectory versus time is depicted in figure 1 . This representation has not been used by other authors who studied the free fall, but it is very interesting, more intuitive and practical. In figure (1), we can clearly identify two stages of the leap's evolution. The first stage, from exit time $t=0$ to $75 \mathrm{~s}$, is a quasi-perfect vertical one dimensional movement. The second stage from $t=75$ to $t=260 \mathrm{~s}$ (main chute deployment, at 18:10:52.0 UTC) looks more like a classic free fall of a skydiver who does not try to increase his speed but instead tries to fall slowly and steadily in a nice regular "belly-to-earth" position. In most papers [4,5 and 8], the acceleration due to gravity is taken as constant, but the change of $\mathrm{g}$ can be easily considered by using the world geodetic system ellipsoidal gravity formula (WGS 1984) with the first-order free-air correction factor:

$$
g(z, \varphi)=9.7803 \frac{1+0.00193 \cdot \sin ^{2}(\varphi)}{\sqrt{1-0.00669 \cdot \sin ^{2}(\varphi)}}-3.086 \times 10^{-6} \cdot z
$$

at the origin of local ENU system $\varphi=33.3408417^{\circ}$

$$
g(z, \varphi)=9.7959-3.086 \times 10^{-6} \cdot z
$$

For $z$ varying from the initial position to the main chute deployment, $2566.8 \leq z \leq 38969.4 \mathrm{~m}$, the acceleration due to gravity is $9.6756 \leq g \leq 9.7879 \mathrm{~m} / \mathrm{s}^{2}$, that is a relative error of $1 \%$ if $g$ is taken as a constant. The total mass of Baumgartner taken for the simulations is $m=121.2 \mathrm{~kg}$ [7].

For this experience, the Red Bull Stratos team did everything to ensure the safety of Baumgartner's jump $[1 ; 10]$. Various measures have been taken, analyzed and processed by the team to validate several world records, and now they are available to us. Baumgartner was equipped with several GPS apparatuses, and in particular a Garmin 18X-5 WAAS GPS with a sampling frequency of 5 position measurements per second. The data files made available also contain temperature, air pressure, speed of sound and triaxial acceleration data. Baumgartner was equipped with a triaxial accelerometer positioned at chest level in order to understand and analyze the environmental stressors experienced [11].

Atmospheric density and speed of sound $\left(M a c h_{1}\right)$ profiles are estimated based on the pressure $P(z)$ and temperature $T(z)$ measurements with:

$$
\rho(z)=\frac{P(z)}{R_{S} T(z)} \text { and } \operatorname{Mach}_{1}(z)=\sqrt{1.40 \cdot R_{S} T(z)} \quad \text { (6 and 7) }
$$



with $R_{S}=287.0 \mathrm{~J} /(\mathrm{K} \mathrm{kg})$. The profiles of temperature, pressure, density and speed of sound are depicted in figure 2 (red curve) and are compared to two classical models, the US Standard atmosphere, 1976 [12] and NRLMSISE-00 Atmosphere Model [13, 14], and to the sounding operated by the Red Bull Stratos team at the Santa Teresa observatory and Roswell airport. The most important data for studying the free fall is for $20 \leq z \leq 40 \mathrm{~km}$. In this part, we have good correlation between the data and the NRLMSISE-00 Atmosphere Model. In the later simulations we will use the data depicted on the red curve. To our knowledge, the data was obtained by the Red Bull Stratos team with a numerical weather forecast model.

For the drag coefficient and the projected area, we use the relations proposed by Guerster and Walter [7]:

$$
\begin{array}{ll}
C_{D}=A & \text { for } M a=\frac{v_{z}}{M a c h_{1}} \leq 0.6 \\
C_{D}=A+B(M a-0.6)^{2} & \text { for } 0.6 \leq M a \leq 1.1 \\
C_{D}=A+0.25 \cdot B-C(M a-1.1) & \text { for } 1.1 \leq M a
\end{array}
$$

and

$$
A_{\perp}(\beta)=A_{x} \sin (\beta)+A_{z} \cos (\beta)
$$

with $A_{x}=1.19 \mathrm{~m}^{2}$ the effective front area and $A_{z}=0.525 \mathrm{~m}^{2}$ the effective top area [7], A, B and C three parameters that should be estimated in the inverse problem.

The last needed information, and probably the most difficult to obtain is the angle of attack $\beta$ during the leap. Guerster and Walter [7], estimate an angle of attack $\alpha=\frac{\pi}{2}-\beta$ with the full video from Baumgartner's point of view during the leap. In this study, the angle of attack is estimated based on the triaxial acceleration measurements (fig.3). Accelerometers are sensitive to both linear acceleration and the local gravitational field. In the absence of linear acceleration, the accelerometer output is a measurement of the rotated gravitational field vector and can be used to determine the accelerometer orientation angles. At the start of a fall, the vector sum of acceleration will tend toward $0 \mathrm{~g}$ (fig 3 ) this is called the phenomenon of weightlessness. Until $t=25 \mathrm{~s}$, the longitudinal and lateral accelerations do not change, after that time and until t=75 s, these accelerations fluctuate a lot. During this time, Baumgartner was in a dangerous situation of spinning, which has been studied in detail by Garbino et al. [11]. The vertical acceleration has a different signal: it changes gradually, probably due to the drag force's module and direction acting on the body (fig. 3). Even if we aren't in a perfectly static situation, and we also neglect the lateral acceleration $A_{y}$, and if we suppose likewise that Baumgartner didn't rotate around his body's z-axis (head first), the angle of attack is estimated with [15, eq. 55 page 19]:

$$
\beta=\cos ^{-1}\left(\frac{A_{z}}{\sqrt{A_{x}^{2}+A_{z}^{2}}}\right)
$$


with $A_{z}$ and $A_{x}$ representing the vertical and longitudinal acceleration respectively. In order to smooth the signal and remove potential linear acceleration, a rolling mean of $2.5 \mathrm{~s}$ is applied to the angle and used later in a linear interpolation lookup table for the simulations (fig. 4).

\section{The numerical implementation}

The programming language we use is Python, a language which gives readable code that closely resembles the equations. The equation (3) is a system of two first-order equations that can be solved by numerical integration. There are a lot of numerical tools called "ODE solver" (ordinary differential equation) to solve this kind of equation [16]. Here, we have chosen to work with the SciPy ecosystem [17], and in particular with the module scipy.integrate.solve_ivp. This module allows us to choose between all the classical numerical integration methods, and trigger events to stop the integration time if needed. By default, we select the Runge-Kutta method. We can also set the maximum allowed time step size during integration, which allows us to properly control any variations in the parametric functions, like the angle of attack. At this stage, we have all the necessary information allowing us to program and to solve the direct problem, apart from the parameters $A, B$ and $C$ appearing in the drag force. A useful solution is to generate uniformly distributed random parameters between bounds and/or to take some values from literature and to build the code and do tests. This is an important step before tackling the inverse problem, since the direct code must be solved in as little CPU time as possible, and be reliable and robust regardless of the parameters used.

\section{The inverse problem}

The inverse problem covers a very wide range of formulations and fields of application. In this part, we will present an application of a classical non-linear inverse problem. Here, the aim is to find the model parameters we don't know that produce the data we have measured. From a mathematical point of view, we formulate this with the help of a classical cost function or objective function, i.e. the mean sum square error between model output and the measures:

$$
O\left(p ; y, y_{m e s}\right)=\frac{1}{n m-n p} \sum_{i=1}^{n n}\left(y_{i}(p)-y_{i}^{*}\right)^{2}
$$

with $p=(A, B, C)$ the vector of parameters, $\mathrm{nm}$ and $\mathrm{np}$ the number of measurements and parameters respectively, $y_{i}(p)$ the model output, e.g. the speed and $y_{i}^{*}$ the measurements. The aim is to find the parameters that minimize the objective function, which is done iteratively. Generally, the algorithm starts with a set of initial parameters and calculates the corrections to be made to the parameters, so that the objective function decreases until a criterion of the objective function is reached and/or until the parameter values don't change anymore. There are plenty of algorithms that can be used to minimize a function. The most widely used is probably the Levenberg-Marquardt algorithm. The choice of a good algorithm is problem dependent, on the number of parameters, the bounds on the 
parameters, the number of available measurements, the correlations between parameters, the structure of the model and so forth. We can distinguish two main classes of algorithms: global and local algorithms. According to the author's own experience, the most efficient strategy when solving a minimization problem is to be able to use several kind of algorithms and analyze the different results. Here, we have chosen to use LMFIT: Non-Linear Least-Squares Minimization and Curve-Fitting for Python [18]. This library allows us to use more than twenty methods of minimization, from the local Levenberg-Marquardt least squares method to the Markov Chain Monte Carlo (MCMC) algorithm, amongst others. A complete discussion of inverse methods is beyond the scope of this document; the aim here is simply to give students access to up-to-date methods and algorithms as "gray-box", and not completely "black-box".

The use of Imfit.minimize() module is straightforward, as long as the user starts with one of the numerous examples available on the GitHub repository ( https://Imfit.github.io/Imfitpy/examples/index.html) and adapts it to their problem. Principally, the user has to build a callable function that corresponds to the direct problem. This function should return the difference between model and measure, i.e. the residual vector:

$$
\varepsilon_{i}=y_{i}(p)-y_{i}^{*}
$$

In our example, the state variable of interest is the speed. $y_{i}(p)$ is the solution of the system of equation (3), i.e. $v_{z}\left(t=t_{i}\right)$, with $t_{i}$ the discrete time observations for $i=1 \cdots n m$. In the local ENU coordinate system, we can compute the speed by using the evolution of the altitude $z$ over time with a first order finite difference equation:

$$
y_{i}^{*} \equiv v_{i} \simeq \frac{z_{i+1}\left(t_{i+1}\right)-z_{i}\left(t_{i}\right)}{t_{i+1}-t_{i}}
$$

The GPS used has a sampling frequency of 5 position measurements per second; for the whole duration of the leap (from $t=0$ to 260 s), this gives us a total number of available measurements of 1300 . In figure (1), we have seen that the vertical free fall is well approximated during only the first part of the leap, that is for a period from $t=0$ to $75 \mathrm{~s}$. For the parameter estimation, we only use the data obtained during this first part of the leap and with one measurement per second, that is a total number of measurements $n m=75$, for a total number of parameters to be estimated $n p=3$.

\section{Results and discussion}

In order to start the inverse problem, it is often necessary to have some prior knowledge of the parameters we want to estimate. Sometimes, these parameters have no physical meaning, but even in this case we should give an initial set of parameters and some lower and upper bound values (Table 1). For this simulation, we use the trust region reflective method to minimize the objective function 
[18]. At the end of the estimation, after 11 iterations, the final set of parameters and the $95 \%$ confidence interval on the parameters are:

$$
\begin{gathered}
A=0.617+/-0.035(5.7 \%) \\
B=0.840+/-0.259(30.8 \%) \\
C=1.425+/-0.344(24.2 \%)
\end{gathered}
$$

The results differ from those of Guerster and Walter [7] for the parameters B and C. We find slightly larger values for almost identical confidence intervals. It should be noted that the parameters $A$ and $B$ have a correlation of -0.92 and for B and C of 0.89 . Since we didn't use exactly the same angle of attack as Guerster and Walter [7], it is possible to find different parameters that give almost identical results for the speed. This is a key point for parameter estimation, as there is not always a unique solution to the inverse problem, but instead depends greatly on the model structure and on available data. The drag force in the model depends on the product of the drag coefficient and the projected area, so this creates a perfect correlation between them. Colino and Barbero's [6] strategy was to use the product and take different values of this product depending on the stages of the leap. The results of the inverse problem for the altitude, speed and Mach number are depicted in figure (5). We can observe a fairly good match between the measurements and the modelling. The gray region (fig. 5) represents the $95 \%$ confidence interval of the estimated speed. In the second stage, from $t=75$ to $t=260 \mathrm{~s}$, we observe larger differences between the measurements and the model, probably due to a 3D effect on the trajectory. A more detailed graphic concerning the supersonic stage is given in figure (6). We found a total supersonic time of 30s, a maximum speed, reached at $\mathrm{t}=50 \mathrm{~s}, v=375.7 \pm 10.8 \mathrm{~m} / \mathrm{s}$ and a Mach number $M a=1.25 \pm 0.03$. We can also see the impact of variations over time of the angle of attack on the speed, for $\mathrm{t}=40 \mathrm{~s}$ and $\mathrm{t}=74 \mathrm{~s}$.

Now that the whole model has been built (direct and inverse), it is relatively simple to carry out additional tests if needed, for example to test other sets of initial parameters, or to assume that $g$ is constant, or to propose a different model for the drag coefficient or for the air density equation, or to modify the time parametric function for the angle of attack.

If one wishes to have more reliable information on the parameters and their uncertainties, it is possible with LMFIT to use an MCMC algorithm to determine the posterior distributions for the parameters, and not only a local uncertainty estimation.

\section{Conclusion}

The good thing about building a full model, i.e. direct and inverse, is that it gives students the opportunity to learn multiple skills by discussing the problem on the conceptual level, together with mathematical formulation, finding numerical methods, appropriate algorithms and writing the computer code, and testing the programs and using them to perform numerical experiments, to 
analyze the problem, and to compare the results to real data. All of this is now possible, especially with Python if we agree to use some libraries as a "gray-box" and explain the principles of these algorithms to students. Additionally, representing the computational results graphically is always very important, and this is facilitated for students with Python [19]. Finally, teaching the building of an inverse problem has a lot of similarities with creating an Investigative Science Learning Environment [20, figure 1.3 page 1-7 ].

\section{Acknowledgments}

A special thanks to Jonathan Clark and Alex Garbino, Department of Medicine, Section of Emergency Medicine and Center for Space Medicine, Baylor College of Medicine, Houston - TX, for support and making the flight data available to us. A sincere thank you to our colleague, Rob Simmons, from Institut Universitaire de Technologie Louis Pasteur, for his diligent proofreading of the article.

\section{References:}

[1] Red Bull Stratos www.redbullstratos.com.

[2] Red Bull Stratos: Full Scientific Data Report, Findings of the Red Bull Stratos Scientific Summit, California Science Center, Los Angeles, California, USA January 23, 2013, https://issuu.com/redbullstratos/docs/red bull stratos summit report final 050213.

[3] Samsonau S. V.,2018. Computer simulations combined with experiments for a calculus-based physics laboratory course, Phys. Educ. 53055013.

[4] Benacka J., 2010. High-altitude free fall revised. American Journal of Physics 78(6)616-619; doi: 10.1119/1.3298375.

[5] Theilmann F. and Apolin M., 2013. Supersonic freefall-a modern adventure as a topic for the physics class. Phys. Educ. 48(2)150-158.

[6] Colino J. M. and Barbero A. J., 2013. Quantitative model of record stratospheric freefall. Eur. J. Phys. (34)841-848, doi:10.1088/0143-0807/34/4/841.

[7] Guerster M. and Walter U., 2017. Aerodynamics of a highly irregular body at transonic speedsAnalysis of STRATOS flight data. PLoS ONE 12(12): e0187798; doi: 10.1371/journal.pone.0187798. 
[8] Corvo T., 2019. An Analytical Solution to the Extreme Skydiver Problem. The Physics Teacher 57:287-289; doi: 10.1119/1.5098912.

296

297

298

299

300

301

302

303

304

305

306

307

308

309

310

311

312

313

314

315

316

317

318

319

320

321

322

323

324

325

326

327

[9] Barger V. and Olsson M., 1995. Classical Mechanics: A Modern Perspective, Second Edition, McGraw-Hill, New York, 418pp.

[10] Blue R.S., Law J., Norton S.C., Garbino A., Pattarini J. M., Turney M. W., Clark J.B., 2013. Overview of medical operations for a manned stratospheric balloon flight. Aviat Space Environ Med (84)237-41.

[11] Garbino A., Blue R.S., Pattarini J. M., Law J., Clark J.B., 2014. Physiological monitoring and analysis of a manned stratospheric balloon test program. Aviat Space Environ Med (85)177-82.

[12] U.S. standard atmosphere, 1976, Washington, D.C., NOAA--S/T 76-1562,241pp.

[13] J.M. Picone, A.E. Hedin, D.P. Drob, and A.C. Aikin, 2002. NRLMSISE-00 empirical model of the atmosphere: Statistical comparisons and scientific issues, J. Geophys. Res., 107(A12), 1468, doi:10.1029/2002JA009430.

[14] Papitashvili N. 2001. Web interface for NRLMSISE-00 Atmosphere Model, The Community Coordinated Modeling Center, https://ccmc.gsfc.nasa.gov/modelweb/models/nrlmsise00.php.

[15] Pedley M., 2013. Tilt Sensing Using a Three-Axis Accelerometer. Freescale Semiconductor, Inc. AN3461 Application Note Rev. 6, 22pp.

[16] Linge S. and Langtangen H. S., 2020. Programming for Computations - Python : A Gentle Introduction to Numerical Simulations with Python 3.6, Second Edition, Springer Open, 350pp. https://doi.org/10.1007/978-3-030-16877-3.

[17] Pauli Virtanen, Ralf Gommers, Travis E. Oliphant, Matt Haberland, Tyler Reddy, David Cournapeau, Evgeni Burovski, Pearu Peterson, Warren Weckesser, Jonathan Bright, Stéfan J. van der Walt, Matthew Brett, Joshua Wilson, K. Jarrod Millman, Nikolay Mayorov, Andrew R. J. Nelson, Eric Jones, Robert Kern, Eric Larson, CJ Carey, İlhan Polat, Yu Feng, Eric W. Moore, Jake VanderPlas, Denis Laxalde, Josef Perktold, Robert Cimrman, Ian Henriksen, E.A. Quintero, Charles R Harris, Anne M. Archibald, Antônio H. Ribeiro, Fabian Pedregosa, Paul van Mulbregt, and SciPy 1.0 Contributors. (2020) SciPy 1.0: 
328 Fundamental Algorithms for Scientific Computing in Python. Nature Methods, 329 https://doi.org/10.1038/s41592-019-0686-2.

330

331

[18] Newville M., Stensitzki T., Allen D. B. and Ingargiola A., 2014. LMFIT: Non-Linear Least-Square

332

Minimization and Curve-Fitting for Python (Version 1.0.1). Zenodo.

333

http://doi.org/10.5281/zenodo.11813.

334

335 [19] Weber J. and Wilhelm Th., 2020. The benefit of computational modelling in physics teaching: a 336 historical overview, Eur. J. Phys.41 034003.

337

338 [20] Etkina E., Brookes, D. T. and Planinsic G., 2019. Investigative Science Learning Environment. When

339 learning physics mirrors doing physics. Morgan \& Claypool Publishers, 138pp.

340 http://dx.doi.org/10.1088/2053-2571/ab3ebd.

341 
342

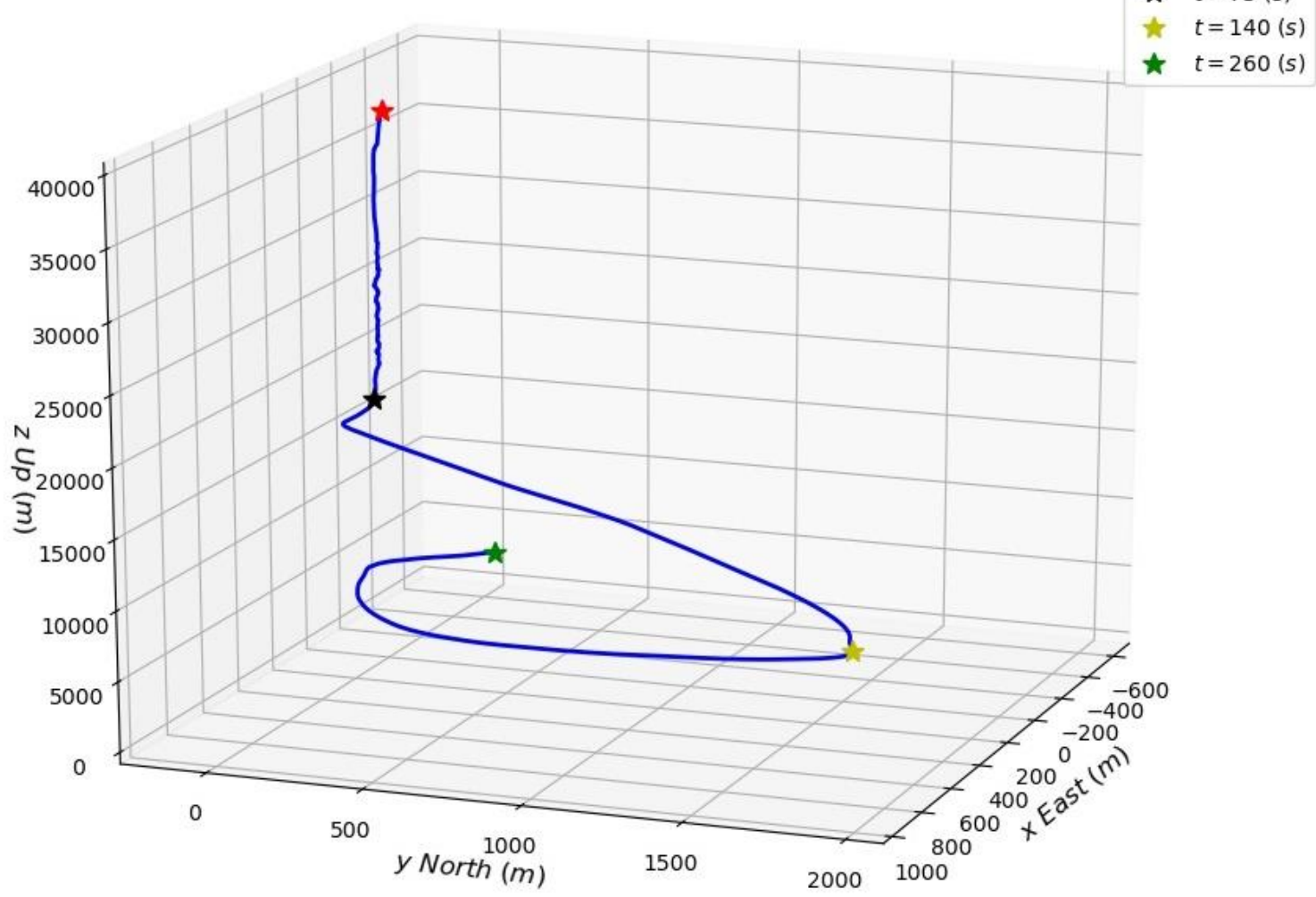

Figure 1: Skydiver position evolution through the leap, expressed in a local tangent plane coordinates system, as origin $\left(\varphi=33.3408417^{\circ}, \lambda=-103.7679067^{\circ}, z=0.0 \mathrm{~m}\right)$
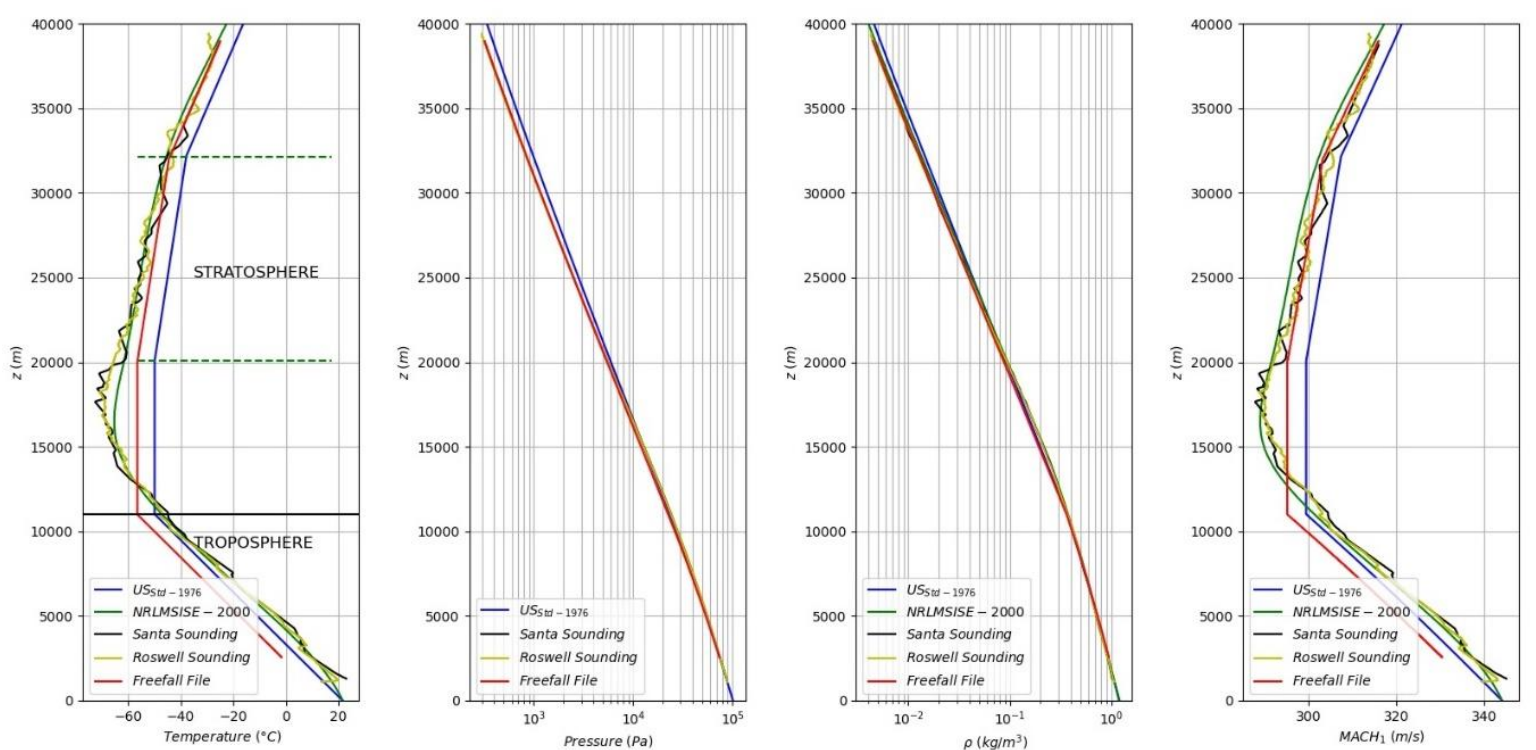

Figure 2: Comparison of the different atmospheric data; the red curves are used for the simulations. 

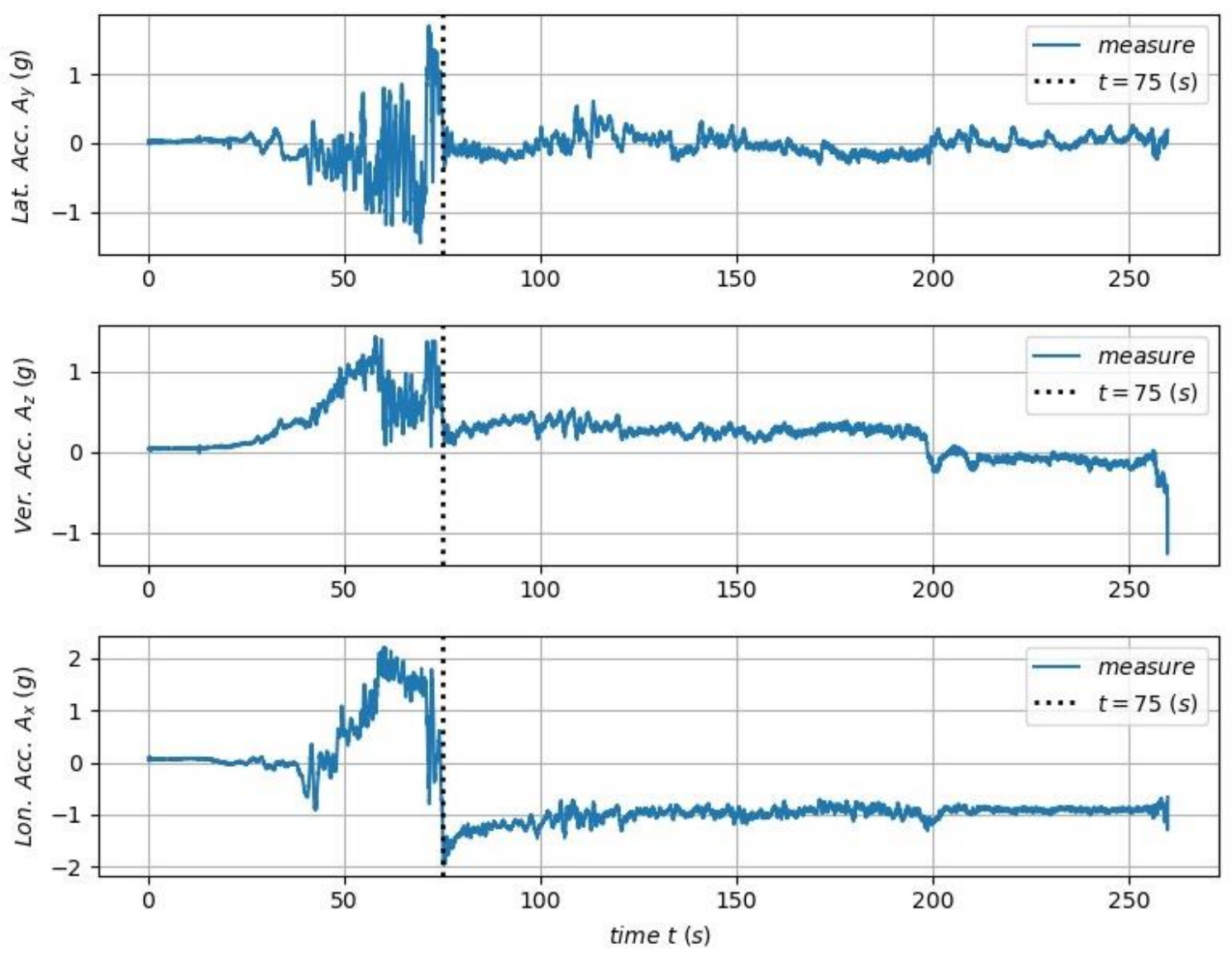

Figure 3: Measured triaxial acceleration

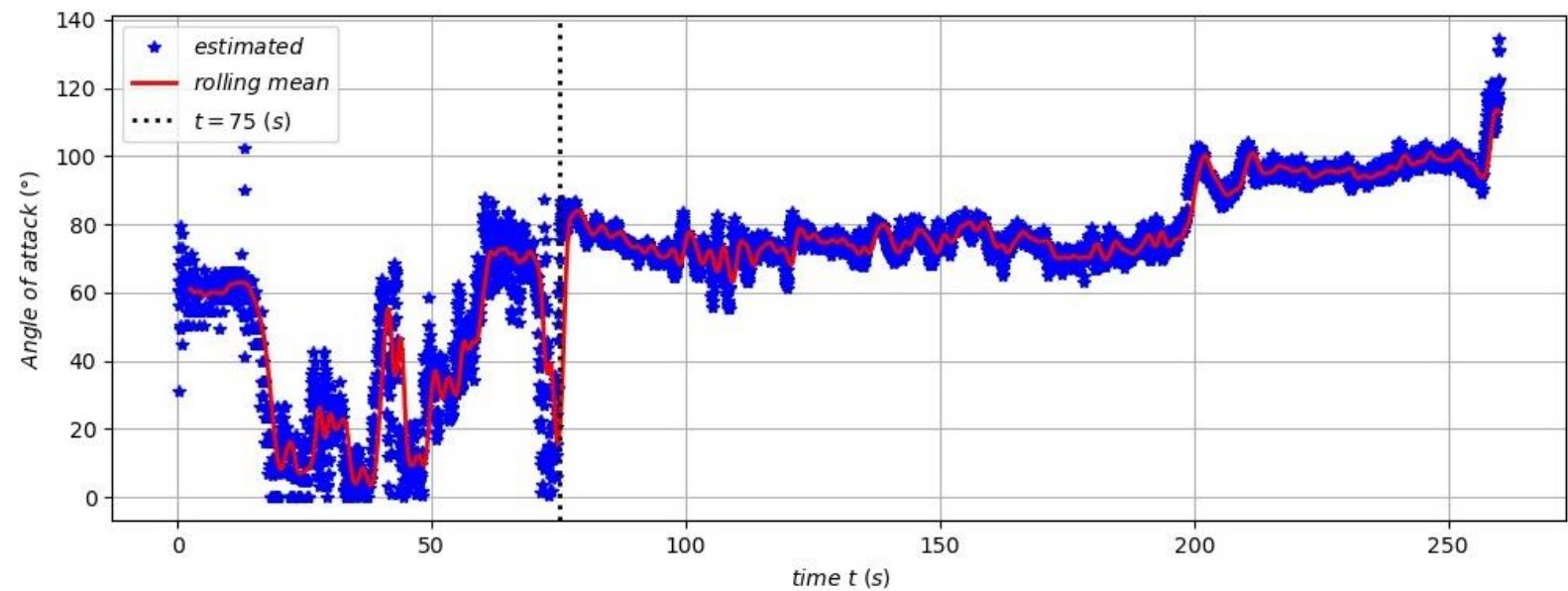

Figure 4: Estimated angle of attack based on the acceleration $A_{x}$ and $A_{z}$ 
Table 1: Initial set of parameters with lower and upper bounds.

\begin{tabular}{cccc}
\hline Parameters $p_{k}$ & Min value & Initial value & Max value \\
\hline $\mathrm{A}$ & 0.25 & 0.6 & 2 \\
$\mathrm{~B}$ & 0.1 & 0.6 & 2 \\
$\mathrm{C}$ & 0 & 0.3 & 5 \\
\hline
\end{tabular}

354

355
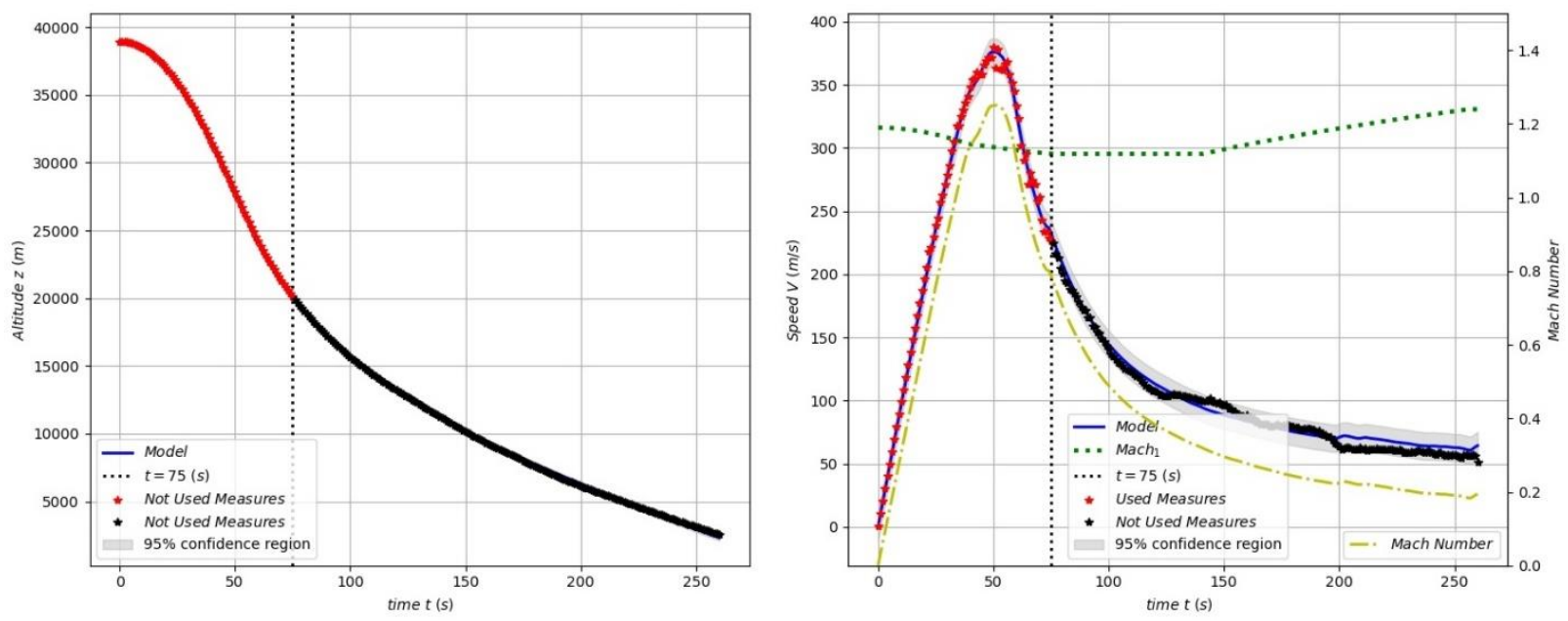

Figure 5: Measured and predicted altitude, speed and Mach number with the final set of parameters.

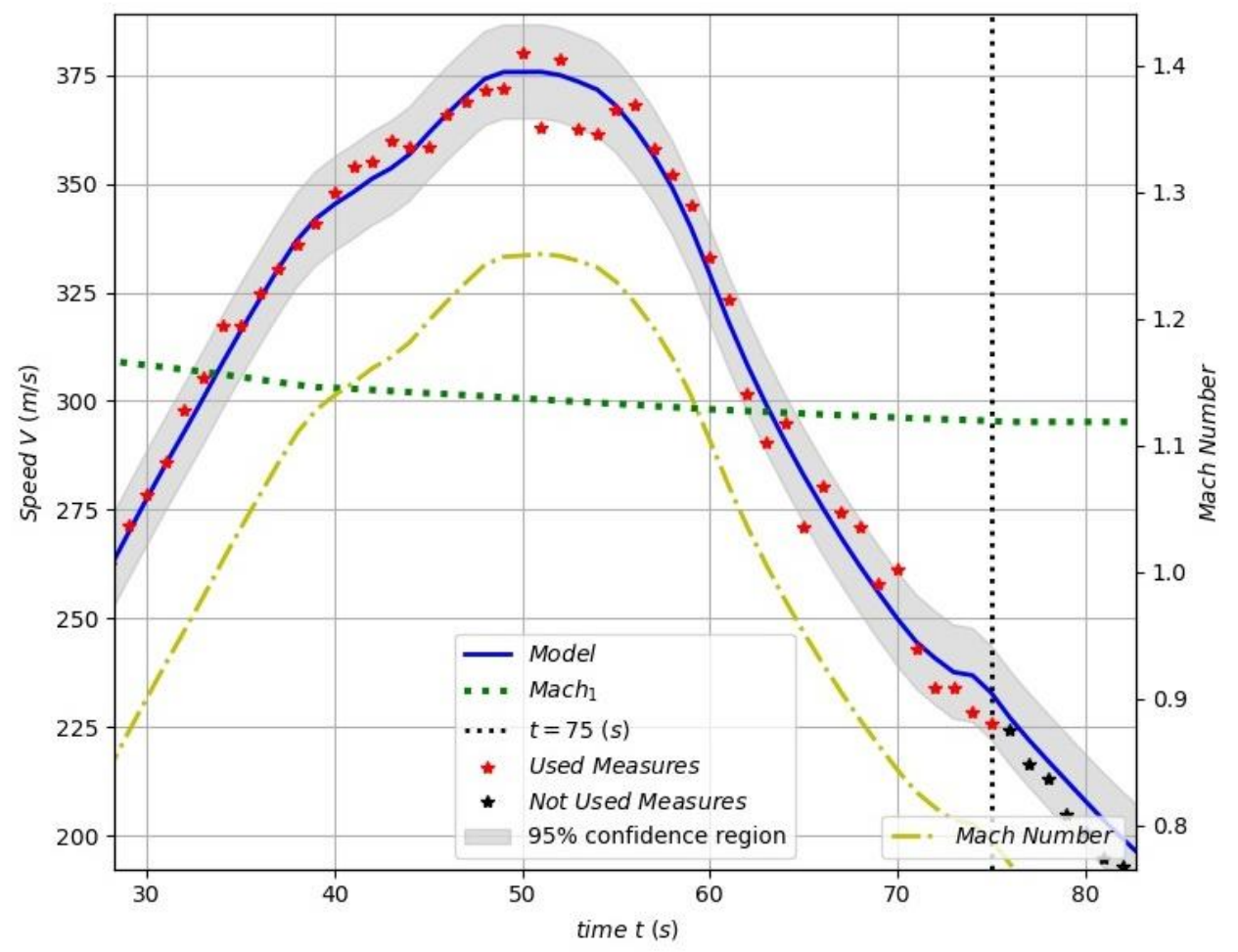

Figure 6: Measured and predicted altitude, speed and Mach number with the final set of parameters 